\title{
Shedding light on the highest energy emission from GRBs with MAGIC observations
}

\author{
Alessio Berti, ${ }^{a, *}$ Zeljka Bosnjak, ${ }^{b}$ Stefano Covino, ${ }^{c}$ Satoshi Fukami, ${ }^{d}$ Susume \\ Inoue ${ }^{e}$ Francesco Longo, ${ }^{f, g}, h$ Davide Miceli, ${ }^{g, i, j}$ Razmik Mirzoyan, ${ }^{a}$ Elena Moretti, ${ }^{k}$ \\ Lara Nava, ${ }^{g}, h, l$ Koji Noda, ${ }^{d}$ David Paneque, ${ }^{a}$ Antonio Stamerra, ${ }^{l}$ Yusuke Suda $^{m}$ and \\ levgen Vovk $^{d}$ on behalf of the MAGIC Collaboration ${ }^{\dagger}$ \\ ${ }^{a}$ Max Planck Institute for Physics, Föhringer Ring 6, Munich, Germany \\ ${ }^{b}$ Faculty of Electrical Engineering and Computing, University of Zagreb, Zagreb, Croatia \\ ${ }^{c}$ INAF, Osservatorio Astronomico di Brera, Merate, Italy \\ ${ }^{d}$ Institute for Cosmic Ray Research, The University of Tokyo, Kashiwanoha 5-1-5, Kashiwa, Japan \\ ${ }^{e}$ RIKEN, Hirosawa 2-1, Wako, Japan \\ ${ }^{f}$ University of Trieste, Department of Physics, via Valerio 2, Trieste, Italy \\ ${ }^{g}$ INFN, Sezione di Trieste, via Valerio 2, Trieste, Italy \\ ${ }^{h}$ Institute for Fundamental Physics of the Universe (IFPU), via Beirut 12, Trieste, Italy \\ ${ }^{i}$ University of Udine, via delle Scienze 206, Udine, Italy \\ ${ }^{j}$ now at Laboratoire d'Annecy de Physique des Particules (LAPP), CNRS-IN2P3, 9 Chemin de Bellevue - \\ BP 110, 74941 Annecy Cedex, France \\ ${ }^{k}$ Institut de Física d'Altes Energies (IFAE), Edifici Cn, Bellaterra (Barcelona), Spain \\ ${ }^{l}$ National Institute for Astrophysics (INAF), I-00136 Rome, Italy \\ ${ }^{m}$ Physics Program, Graduate School of Advanced Science and Engineering, Hiroshima University, \\ 739-8526 Hiroshima, Japan \\ E-mail: aberti@mpp.mpg.de
}

\footnotetext{
${ }^{*}$ Presenter

${ }^{\dagger}$ a complete list of the MAGIC Collaboration authors can be found at the end of the proceedings
} 
On 14th January 2019, the MAGIC collaboration achieved the first significant detection at $\mathrm{TeV}$ energies of a gamma-ray burst (GRB), namely GRB 190114C. This observation sets the first experimental proof of very high energy (VHE, $>100 \mathrm{GeV}$ ) gamma-ray emission in GRBs, after more than 50 years from the first GRB detection and many searches with Cherenkov telescopes in the last decades. The data collected by MAGIC and by more than 20 other ground-based and spaceborne instruments, spanning 17 orders of magnitude in energy, revealed a new GeV-TeV emission component in the GRB afterglow. This unprecedented multi-wavelength dataset, including VHE data for the first time, allowed a detailed study of the broadband emission. A one-zone synchrotronself Compton scenario with internal $\gamma-\gamma$ absorption could be used to describe the broadband emission, using parameters compatible with those found in previous studies of GRB afterglows below the GeV energy range. This detection opened a new era in the studies of GRBs, leading to new questions such as the universality of $\mathrm{TeV}$ emission in different types of GRBs. In this contribution, we will present the GRB follow-up program performed by the MAGIC collaboration, which started more than 15 years ago. We will highlight the results on GRB 190114C, discuss the implications for GRB physics, and report the latest developments and the prospects for future observations of GRBs with the MAGIC telescopes.

$37^{\text {th }}$ International Cosmic Ray Conference (ICRC 2021)

July 12th - 23rd, 2021

Online - Berlin, Germany 


\section{Introduction}

Gamma-ray bursts (GRBs) are transient sources routinely observed as bright flashes of gammaray radiation, the so called prompt emission, which is characterized by rapid flux variability down to millisecond scales. This first emission phase is usually very brief, lasting from fractions of a second to hundreds of seconds. According to its duration, GRBs are classified as long and short, with $2 \mathrm{~s}$ as separation value. The prompt phase is followed, with partial overlap in time, by the afterglow, a long lasting (from days to weeks, in some cases months) but fainter emission decreasing with time. Differently from the prompt, the afterglow has been detected at different wavelengths, from radio to $\mathrm{GeV}$ gamma rays.

GRBs are the result of catastrophic events involving compact objects, such as the explosion of massive stars or the merger of binary systems of neutron stars. These events lead to the birth of black holes or neutron stars, from which collimated jets of plasma can be ejected at ultrarelativistic velocities. In the inner regions of the jet, the gamma rays of the prompt phase are produced via a not-yet-well-established mechanism (e.g. synchrotron radiation, magnetic reconnection). The jet will eventually interact with the surrounding medium, generating shock waves (in jargon they are called external shocks) where particles are accelerated and emit radiation. Within this picture, the broadband afterglow emission from radio to $\mathrm{GeV}$ gamma rays is well explained as synchrotron radiation produced by electrons accelerated at the external shocks.

In recent observations, hints of a possible new component in the afterglow were reported in a few GRBs detected by the Fermi-LAT instrument. Driven also by theoretical models, the presence of such additional component can be proved by observations in the very high energy range (VHE, $E \gtrsim 100 \mathrm{GeV}$ ) performed with Imaging Atmospheric Cherenkov telescopes (IACTs). A detection at such high energies can provide invaluable information about the particle acceleration processes and jet dynamics. The observations of GRBs with IACTs has been historically challenging because of both physics and technical reasons, such as the strong flux absorption at VHE due to the extragalactic background light (EBL), or the delay in the observation needed to repoint the telescopes. Overcoming these challenges, here we report the first detection at $\mathrm{TeV}$ energies of the long GRB 190114C achieved by the MAGIC telescopes. For more details we refer to [1] and [2].

\section{MAGIC observation and detection of GRB 190114C}

The MAGIC telescopes are two twin Cherenkov telescopes of $17 \mathrm{~m}$ diameter located at $2200 \mathrm{~m}$ above sea level in the observatory on the Roque de Los Muchachos mountain in La Palma, Canary Islands, Spain. As typical for IACTs, they have a limited field of view, around $3.5^{\circ}$ for MAGIC. The detection of GRBs is one of the primary scientific targets of MAGIC. The low energy threshold $(\sim 50 \mathrm{GeV})$, the high sensitivity at sub-TeV energies and the fast slewing speed $\left(7^{\circ} \mathrm{s}^{-1}\right.$ in the so called fast mode) are key parameters for such goal. To reduce the latency and issues in performing GRB observations, MAGIC implemented a fully automatic reaction to GRB alerts received from the Gamma-ray Coordinates Network (GCN; https://gcn.gsfc . nasa.gov/) through its automatic alert system (AAS, see [1]).

On 14th January 2019, at 20:57:03 UT (hereafter $T_{0}$ ), the Swift-BAT and Fermi-GBM instruments detected GRB 190114C, accompanied also by a bright optical counterpart. GRB 190114C 


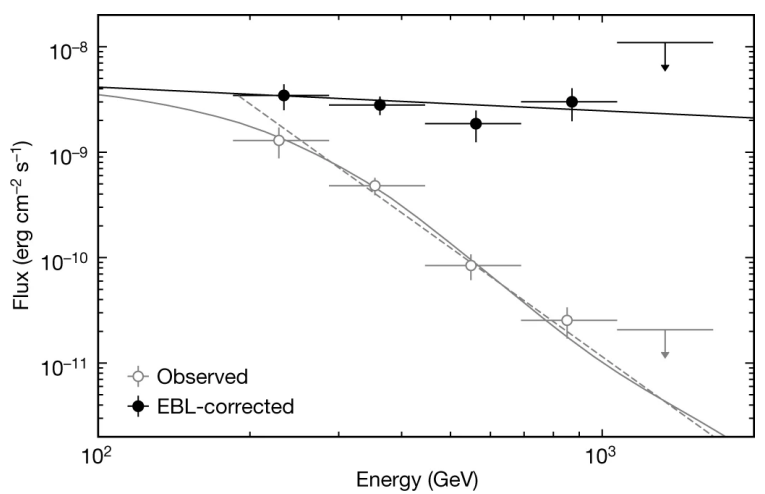

Figure 1: Observed (grey open circles) and intrinsic (blue filled circles) time integrated spectra of GRB 190114C between $T_{0}+62 \mathrm{~s}$ and $T_{0}+2454 \mathrm{~s}$. The best fit power-law function to the intrinsic spectrum is represented by the thin black solid line, while the thin grey line is obtained from such curve after taking into account the EBL attenuation. The grey dashed line is the best fit power-law function to the observed spectrum. Figure extracted from [1].

was later detected by other instruments such as Fermi-LAT, AGILE/MCAL, INTEGRAL/SPI-ACS, Insight/HXMT and Konus-Wind. The optical observations performed by the Nordic Optical Telescope (NOT) and the Gran Telescopio Canarias (GTC) allowed the determination of the redshift, $z=0.425$. According to the duration of the burst, GRB 190114C is classified as a long GRB, confirmed by the detection of an associated supernova component about 15 days after $T_{0}$.

MAGIC received the alert containing the coordinates of GRB 190114C provided by Swift-BAT at 20:57:25 UT $\left(T_{0}+22 \mathrm{~s}\right)$, triggering the automatic follow-up procedure. The telescopes started tracking the source at $T_{0}+50 \mathrm{~s}$, while the data taking started at $T_{0}+57 \mathrm{~s}$, with stable acquisition rates starting from $T_{0}+62 \mathrm{~s}$. The exposure of the observation was $4.12 \mathrm{~h}$, covering a zenith range from $55.8^{\circ}$ to $81.1^{\circ}$. The weather conditions were very good, but due to the presence of the moon, the night sky background was approximately 6 times brighter than the one during dark nights observations, resulting in an higher energy threshold.

Already in the MAGIC real-time analysis, GRB 190114C was detected at the 20 sigma level in the first 20 minutes of data, subsequently reaching 50 sigma (see Extended Data Figure 2 in [1]) in offline analyses that included proper calibrations and a MC tuned to describe the actual performance of MAGIC during these observations.

The time integrated spectrum of GRB $190114 \mathrm{C}$ as observed by MAGIC between $T_{0}+62 \mathrm{~s}$ and $T_{0}+2454 \mathrm{~s}$, after proper unfolding, is shown in Figure 1. This can be described by a simple power law with index $\alpha_{\text {obs }}=-5.34 \pm 0.22$ from $0.2 \mathrm{TeV}$ extending up to $1 \mathrm{TeV}$. Figure 1 also shows the intrinsic spectrum of GRB 190114C, after taking into account the EBL absoprtion expected for the redshift of this GRB. Again, a power law describes well such spectrum, with spectral index $\alpha_{\text {int }}=-2.22_{-0.25}^{+0.23}$, without any evidence for any spectral break or cutoff. Also, the spectrum extends beyond $1 \mathrm{TeV}$ at $95 \%$ confidence level. This is the first time that photons with such energies have been detected from a GRB. This is a remarkable result, given the large EBL absorption, about a factor 300 at $1 \mathrm{TeV}$. Moreover, the intrinsic spectral index close to $\alpha_{\text {int }}=-2$ implies that the energy radiated in the VHE range is considerable, and almost comparable to the one emitted at lower energies. 


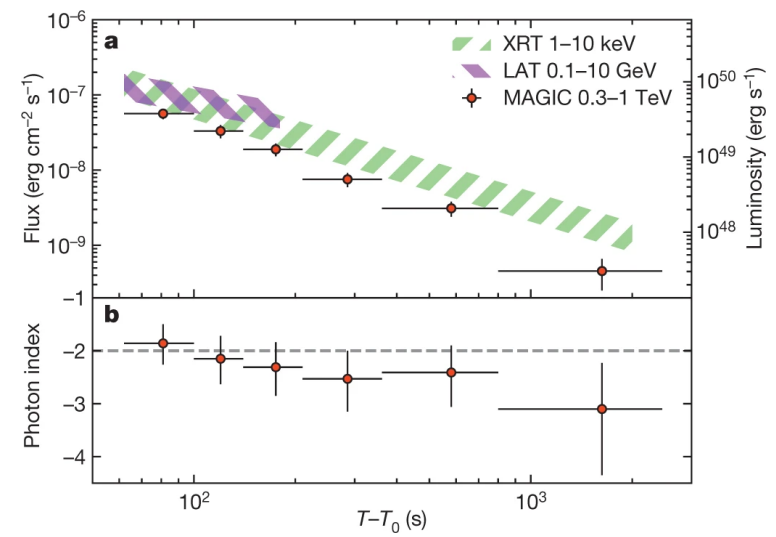

Figure 2: a: energy flux light curve for GRB 190114C by different instruments: MAGIC (red circles), XRT (green band) and LAT (red band). b: temporal evolution of the intrinsic spectral index measured by MAGIC. Figure extracted from [1].

In order to investigate the origin of the detected VHE emission, Figure 2 shows the MAGIC intrinsic energy flux light curve computed between 0.3 and $1 \mathrm{TeV}$ after EBL correction, produced in six time bins covering the first 40 minutes of the observation. The flux measured at $0.3 \mathrm{TeV}$ during the first few seconds was around 100 times higher than the one of the Crab Nebula at the same energy, making GRB $190114 \mathrm{C}$ the brightest source at $0.3 \mathrm{TeV}$. The light curve does not show any variability, and follows a simple temporal power-law decay with index $\beta=1.60 \pm 0.07$. The light curves measured by Fermi-LAT and Swift-XRT in the $0.1-10 \mathrm{GeV}$ and $1-10 \mathrm{keV}$ energy ranges respectively show a similar temporal decay. This supports an afterglow origin for the emission detected by MAGIC. Additionally, temporal and spectral properties as measured by Swift-BAT and Fermi-GBM after $T_{0}+25 \mathrm{~s}$ are typical of afterglow emission, strengthening the same conclusion in the case of MAGIC.

After establishing the afterglow origin of the VHE emission, we investigated the responsible physical process. The similar behavior of MAGIC and XRT light curves points to a close relation between the processes producing the emission in the VHE and X-ray ranges. In the case of GRB 190114C, as for most GRB afterglows, the broadband afterglow emission up to GeV energies can be well described as synchrotron radiation from ultrarelativistic electrons accelerated at the external shock. However, synchrotron photons reach a maximum energy (the so called synchrotron burnoff limit), which depends on the time-dependent bulk Lorentz factor of the GRB jet, $\Gamma_{\mathrm{b}}(t)$, through the relation $E_{\mathrm{syn}, \max } \sim 100\left(\Gamma_{\mathrm{b}}(t) / 1000\right) \mathrm{GeV}$. Figure 3 shows the time-dependent maximum energy of synchrotron photons for two different density profiles for the interstellar medium. Comparing the synchrotron burnoff limit as a function of time with the photon energies measured by MAGIC (blue-scale colored bins), we can conclude that the synchrotron process cannot explain the emission detected in the VHE range, unequivocally proving the presence of a separate component in the afterglow of GRB 190114C, as discussed in the next section. 


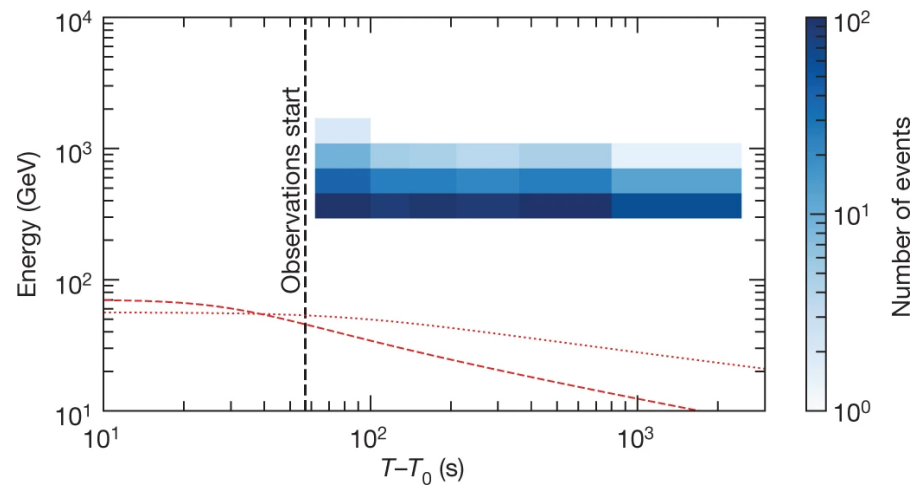

Figure 3: Time and energy distribution of the photons detected by MAGIC. The dotted and dashed curves correspond to the maximal energy of synchrotron photons in the standard afterglow theory for a homogeneous density and wind density profiles respectively. Figure extracted from [1].

\section{A new emission component in the afterglow}

The exceptional brightness of GRB 190114C and its detection by MAGIC triggered an extended multi-wavelength campaign. As shown in Figure 4, the data available for this GRB span 17 orders of magnitude in energy, collected by more than 20 different ground- and space-based instruments. In particular, in the early afterglow phase, there are contemporaneous data available in the keV (XRT, BAT and GBM), GeV (LAT) and TeV (MAGIC) energy ranges. This allows for a temporal spectral analysis of the afterglow emission in order to investigate the physical process underlying the emission in the MAGIC energy range.

As anticipated in section 2, synchrotron radiation cannot explain the energies of the photons detected by MAGIC. The simplest explanation is synchrotron self-Compton (SSC) radiation in the external forward shock. In such process the electrons producing the synchrotron photons can upscatter via the Compton mechanism those photons, increasing their energy and producing a second peak in the spectral energy distribution. In order to assess the viability of SSC as the mechanism producing the $\mathrm{TeV}$ emission, the broadband data from $\mathrm{keV}$ to $\mathrm{TeV}$ energies was modelled within the SSC scenario at the external shock in the GRB afterglow.

Figure 5 presents the result of the SSC modeling for two different time intervals, taking into account internal $\gamma-\gamma$ absorption and scattering in the Klein-Nishina regime for the intrinsic spectrum (blue solid line), and EBL absorption for the observed spectrum (black solid line). This marks the first time that a new component is found in the afterglow of a GRB. Moreover, the SSC process can contribute to the LAT emission at late times (e.g. see extended data Figure 7 in [2]), supporting past hints of an additional component from $\mathrm{GeV}$ observations. Remarkably, the parameters of the modeling are compatible with the values found in past GRB afterglows studies considering data from the radio to the $\mathrm{GeV}$ band. This may indicate that the SSC mechanism could be common in GRB afterglows, as discussed in Section 4.

Finally, other processes were considered in order to explain the $\mathrm{TeV}$ emission. Hadronic processes, like synchrotron radiation from protons, can produce $\mathrm{TeV}$ photons. However the energetic required to reproduce the flux levels measured by MAGIC are orders of magnitude larger than what is typically available in GRBs. Given the relatively fast reaction of MAGIC, it is also interesting to 


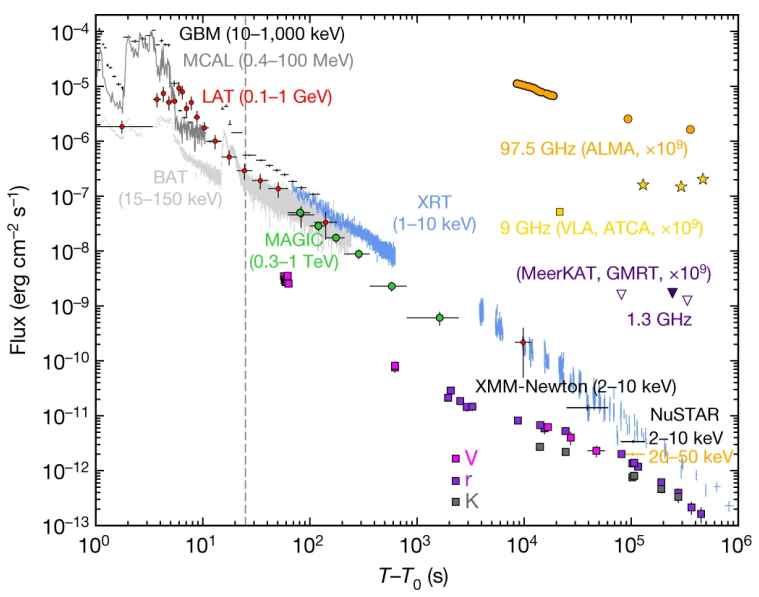

Figure 4: Multiwavelength energy flux light curve of GRB 190114C from radio to gamma rays. The vertical dashed line denotes the approximate end of the prompt phase. Figure extracted from [2].

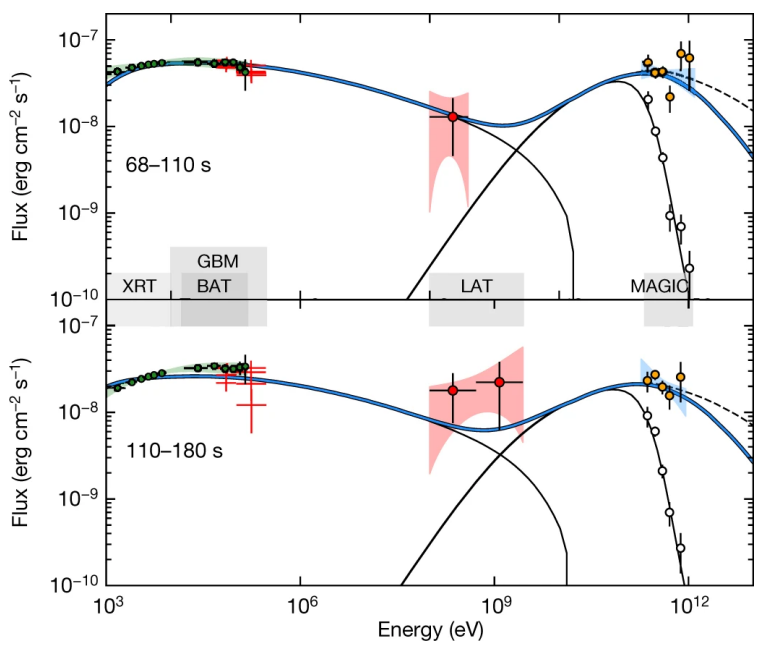

Figure 5: Broadband spectra modelling for two different time intervals in the synchrotron and SSC afterglow scenario. The thick blue curve corresponds to the intrinsic spectrum, the thin black line to the observed spectrum. The dashed black line shows the SSC emission level when $\gamma-\gamma$ internal absorption is neglected. Figure extracted from [2].

investigate if SSC emission from the prompt phase may explain the measured $\mathrm{TeV}$ radiation. In the case of GRB 190114C, it is found that SSC radiation in the prompt may contribute to $\sim 20 \%$ of the observed flux for times $t \lesssim 100 \mathrm{~s}$, favoring the afterglow origin of the $\mathrm{TeV}$ emission.

\section{Prospects for future GRB follow-ups with MAGIC and conclusions}

The detection of a new component in the afterglow of GRB 190114C definitely opened a new era in GRB physics. Nevertheless, such awaited discovery poses new challenges and questions for future GRB follow-ups. First of all, as shown in Section 3, the typical afterglow parameters inferred from the SSC modelling could imply that TeV emission is common in GRB afterglows. Since the properties of GRB 190114C were not exceptional for a long GRB, TeV emission could 
be detected if the distance from the GRB is low enough. This has been demonstrated by other three long GRB detections in the past 3 years: GRB 180720B [3] and GRB 190829A [4] by H.E.S.S. and GRB 201216C [5] by MAGIC. In particular, the first two cases proved that VHE emission can be detected at very late times deep in the afterglow phase. The case of GRB 201216C instead is particularly interesting because of the high redshift of the source, $z=1.1$. It is worth noticing that the VHE gamma-ray emission detected for the three long GRBs that have been recently published (namely GRB 190114C, GRB 180720B and GRB 190829A), can be successfully described through the SSC mechanism.

A second natural question arising from the detection of VHE afterglow emission is whether a similar mechanism may be present in the prompt phase of GRBs. This search is particularly challenging for IACTs, given the intrinsic delay in starting the GRB observations. In this sense, MAGIC fast slewing plays a crucial role in such a quest, e.g. MAGIC could start data taking $24 \mathrm{~s}$ after the burst for GRB 160821B. In the case of long GRBs, this is a promising asset towards the detection of the prompt or prompt-to-afterglow phases.

An additional point concerns short GRBs. Given the strong link with the searches of electromagnetic counterparts from gravitational wave events, the detection of a $\mathrm{TeV}$ component in this class of GRBs may help to further characterize the environment and particle acceleration processes after the merger event. Interesting results in such direction were obtained by MAGIC for the short and nearby $(z=0.16)$ GRB 160821B [6].

Finally, the detection of $\mathrm{TeV}$ photons from distant sources like GRBs opens up the possibility to perform studies related to new physics, such as the search for Lorentz invariance violation (LIV) effects (see e.g. [7]).

Clearly, the VHE era of GRBs has just started. The astonishing results recently achieved are very promising for future follow-ups with MAGIC. This instrument proved to have what it takes in order to achieve the ambitious goal of characterizing GRBs at VHE gamma rays.

\section{Acknowledgements}

We acknowledge the support from the agencies and organizations listed here: https:// magic.mpp.mpg.de/acknowledgments_ICRC2021

\section{References}

[1] MAGIC Collaboration, Nature, 575, 455-458 (2019)

[2] MAGIC Collaboration et al., Nature, 575, 459-453 (2019)

[3] H.E.S.S. Collaboration et al., Nature, 575, 464-467 (2019)

[4] H.E.S.S. Collaboration et al., Science, 372, 1081-1085 (2021)

[5] S. Fukami et al., these proceedings (2021)

[6] V. A. Acciari et al., Astrophysical Journal, 908, 90 (2021)

[7] V. A. Acciari et al. (MAGIC Collaboration), Physical Review Letters, 125, 021301 (2020) 


\section{The MAGIC Collaboration}

V. A. Acciari ${ }^{1}$, S. Ansoldi ${ }^{2,41}$, L. A. Antonelli ${ }^{3}$, A. Arbet Engels $^{4}$, M. Artero ${ }^{5}$, K. Asano $^{6}$, D. Baack ${ }^{7}$, A. Babić ${ }^{8}$, A. Baquero ${ }^{9}$, U. Barres de Almeida ${ }^{10}$, J. A. Barrio ${ }^{9}$, I. Batković ${ }^{11}$, J. Becerra González ${ }^{1}$, W. Bednarek ${ }^{12}$, L. Bellizzi ${ }^{13}$, E. Bernardini ${ }^{14}$, M. Bernardos ${ }^{11}$, A. Berti ${ }^{15}$, J. Besenrieder ${ }^{15}$, W. Bhattacharyya ${ }^{14}$, C. Bigongiari ${ }^{3}$, A. Biland ${ }^{4}$, O. Blanch ${ }^{5}$, H. Bökenkamp ${ }^{7}$, G. Bonnoli ${ }^{16}$, Ž. Bošnjak $^{8}$, G. Busetto ${ }^{11}$, R. Carosi ${ }^{17}$, G. Ceribella ${ }^{15}$, M. Cerruti ${ }^{18}$, Y. Chai ${ }^{15}$, A. Chilingarian ${ }^{19}$, S. Cikota ${ }^{8}$, S. M. Colak ${ }^{5}$, E. Colombo ${ }^{1}$, J. L. Contreras ${ }^{9}$, J. Cortina ${ }^{20}$, S. Covino ${ }^{3}$, G. D’Amico ${ }^{15,42}$, V. D’Elia ${ }^{3}$, P. Da Vela ${ }^{17,43}$, F. Dazzi ${ }^{3}$, A. De Angelis ${ }^{11}$, B. De Lotto ${ }^{2}$, M. Delfino ${ }^{5,44}$, J. Delgado ${ }^{5,44}$, C. Delgado Mendez ${ }^{20}$, D. Depaoli ${ }^{21}$, F. Di Pierro ${ }^{21}$, L. Di Venere ${ }^{22}$, E. Do Souto Espiñeira ${ }^{5}$, D. Dominis Prester $^{23}$, A. Donini ${ }^{2}$, D. Dorner ${ }^{24}$, M. Doro ${ }^{11}$, D. Elsaesser ${ }^{7}$, V. Fallah Ramazani ${ }^{25,45}$, A. Fattorini ${ }^{7}$, M. V. Fonseca ${ }^{9}$, L. Font ${ }^{26}$, C. Fruck ${ }^{15}$, S. Fukami ${ }^{6}$, Y. Fukazawa ${ }^{27}$, R. J. García López ${ }^{1}$, M. Garczarczyk ${ }^{14}$, S. Gasparyan ${ }^{28}$, M. Gaug ${ }^{26}$, N. Giglietto ${ }^{22}$, F. Giordano ${ }^{22}$, P. Gliwny ${ }^{12}$, N. Godinović ${ }^{29}$, J. G. Green ${ }^{3}$, D. Green ${ }^{15}$, D. Hadasch ${ }^{6}$, A. Hahn ${ }^{15}$, L. Heckmann ${ }^{15}$, J. Herrera ${ }^{1}$, J. Hoang ${ }^{9,46}$, D. Hrupec ${ }^{30}$, M. Hütten ${ }^{15}$, T. Inada ${ }^{6}$, K. Ishio ${ }^{12}$, Y. Iwamura ${ }^{6}$, I. Jiménez Martínez ${ }^{20}$, J. Jormanainen ${ }^{25}$, L. Jouvin ${ }^{5}$, M. Karjalainen ${ }^{1}$, D. Kerszberg ${ }^{5}$, Y. Kobayashi ${ }^{6}$, H. Kubo ${ }^{31}$, J. Kushida ${ }^{32}$, A. Lamastra ${ }^{3}$, D. Lelas ${ }^{29}$, F. Leone ${ }^{3}$, E. Lindfors ${ }^{25}$, L. Linhoff ${ }^{7}$, S. Lombardi ${ }^{3}$, F. Longo ${ }^{2,47}$, R. López-Coto ${ }^{11}$, M. López-Moya ${ }^{9}$, A. López-Oramas ${ }^{1}$, S. Loporchio ${ }^{22}$, B. Machado de Oliveira Fraga $^{10}$, C. Maggio ${ }^{26}$, P. Majumdar ${ }^{33}$, M. Makariev ${ }^{34}$, M. Mallamaci ${ }^{11}$, G. Maneva ${ }^{34}$, M. Manganaro ${ }^{23}$, K. Mannheim ${ }^{24}$, L. Maraschi ${ }^{3}$, M. Mariotti ${ }^{11}$, M. Martínez ${ }^{5}$, D. Mazin ${ }^{6,15}$, S. Menchiari ${ }^{13}$, S. Mender ${ }^{7}$, S. Mićanović23, D. Miceli ${ }^{2,49}$, T. Miener ${ }^{9}$, J. M. Miranda ${ }^{13}$, R. Mirzoyan ${ }^{15}$, E. Molina ${ }^{18}$, A. Moralejo ${ }^{5}$, D. Morcuende ${ }^{9}$, V. Moreno ${ }^{26}$, E. Moretti ${ }^{5}$, T. Nakamori ${ }^{35}$, L. Nava ${ }^{3}$, V. Neustroev ${ }^{36}$, C. Nigro $^{5}$, K. Nilsson ${ }^{25}$, K. Nishijima ${ }^{32}$, K. Noda $^{6}$, S. Nozaki ${ }^{31}$, Y. Ohtani ${ }^{6}$, T. Oka ${ }^{31}$, J. Otero-Santos ${ }^{1}$, S. Paiano $^{3}$, M. Palatiello ${ }^{2}$, D. Paneque ${ }^{15}$, R. Paoletti ${ }^{13}$, J. M. Paredes ${ }^{18}$, L. Pavletić ${ }^{23}$, P. Peñil ${ }^{9}$, M. Persic ${ }^{2,50}$, M. Pihet ${ }^{15}$, P. G. Prada Moroni ${ }^{17}$, E. Prandini ${ }^{11}$, C. Priyadarshi ${ }^{5}$, I. Puljak ${ }^{29}$, W. Rhode ${ }^{7}$, M. Ribó ${ }^{18}$, J. Rico ${ }^{5}$, C. Righi ${ }^{3}$, A. Rugliancich ${ }^{17}$, N. Sahakyan ${ }^{28}$, T. Saito ${ }^{6}$, S. Sakurai ${ }^{6}$, K. Satalecka ${ }^{14}$, F. G. Saturni ${ }^{3}$, B. Schleicher ${ }^{24}$, K. Schmidt ${ }^{7}$, T. Schweizer ${ }^{15}$, J. Sitarek ${ }^{12}$, I. Šnidaric ${ }^{37}$, D. Sobczynska ${ }^{12}$, A. Spolon ${ }^{11}$, A. Stamerra ${ }^{3}$, J. Strišković ${ }^{30}$, D. Strom ${ }^{15}$, M. Strzys ${ }^{6}$, Y. Suda ${ }^{27}$, T. Surić ${ }^{37}$, M. Takahashi ${ }^{6}$, R. Takeishi ${ }^{6}$, F. Tavecchio ${ }^{3}$, P. Temnikov ${ }^{34}$, T. Terzić ${ }^{23}$, M. Teshima ${ }^{15,6}$, L. Tosti ${ }^{38}$, S. Truzzi ${ }^{13}$, A. Tutone ${ }^{3}$, S. Ubach ${ }^{26}$, J. van Scherpenberg ${ }^{15}$, G. Vanzo ${ }^{1}$, M. Vazquez Acosta ${ }^{1}$, S. Ventura ${ }^{13}$, V. Verguilov ${ }^{34}$, C. F. Vigorito ${ }^{21}$, V. Vitale ${ }^{39}$, I. Vovk $^{6}$, M. Will ${ }^{15}$, C. Wunderlich ${ }^{13}$, T. Yamamoto $^{40}$, and D. Zarić 29

${ }^{1}$ Instituto de Astrofísica de Canarias and Dpto. de Astrofísica, Universidad de La Laguna, E-38200, La Laguna, Tenerife, Spain ${ }^{2}$ Università di Udine and INFN Trieste, I-33100 Udine, Italy ${ }^{3}$ National Institute for Astrophysics (INAF), I-00136 Rome, Italy ${ }^{4}$ ETH Zürich, CH-8093 Zürich, Switzerland ${ }^{5}$ Institut de Física d'Altes Energies (IFAE), The Barcelona Institute of Science and Technology (BIST), E-08193 Bellaterra (Barcelona), Spain ${ }^{6}$ Japanese MAGIC Group: Institute for Cosmic Ray Research (ICRR), The University of Tokyo, Kashiwa, 277-8582 Chiba, Japan ${ }^{7}$ Technische Universität Dortmund, D-44221 Dortmund, Germany ${ }^{8}$ Croatian MAGIC Group: University of Zagreb, Faculty of Electrical Engineering and Computing (FER), 10000 Zagreb, Croatia ${ }^{9}$ IPARCOS Institute and EMFTEL Department, Universidad Complutense de Madrid, E-28040 Madrid, Spain ${ }^{10}$ Centro Brasileiro de Pesquisas Físicas (CBPF), 22290-180 URCA, Rio de Janeiro (RJ), Brazil ${ }^{11}$ Università di Padova and INFN, I-35131 Padova, Italy ${ }^{12}$ University of Lodz, Faculty of Physics and Applied Informatics, Department of Astrophysics, 90-236 Lodz, Poland ${ }^{13}$ Università di Siena and INFN Pisa, I-53100 Siena, Italy ${ }^{14}$ Deutsches Elektronen-Synchrotron (DESY), D-15738 Zeuthen, Germany ${ }^{15}$ Max-Planck-Institut für Physik, D-80805 München, Germany ${ }^{16}$ Instituto de Astrofísica de Andalucía-CSIC, Glorieta de la Astronomía s/n, 18008, Granada, Spain ${ }^{17}$ Università di Pisa and INFN Pisa, I-56126 Pisa, Italy ${ }^{18}$ Universitat de Barcelona, ICCUB, IEEC-UB, E-08028 Barcelona, Spain ${ }^{19}$ Armenian MAGIC Group: A. Alikhanyan National Science Laboratory, 0036 Yerevan, Armenia ${ }^{20}$ Centro de Investigaciones Energéticas, Medioambientales y Tecnológicas, E-28040 Madrid, Spain ${ }^{21}$ INFN MAGIC Group: INFN Sezione di Torino and Università degli Studi di Torino, I-10125 Torino, Italy 22 INFN MAGIC Group: INFN Sezione di Bari and Dipartimento Interateneo di Fisica dell'Università e del Politecnico di Bari, I-70125 Bari, Italy ${ }^{23}$ Croatian MAGIC Group: University of Rijeka, Department of Physics, 51000 Rijeka, Croatia ${ }^{24}$ Universität Würzburg, D-97074 Würzburg, Germany ${ }^{25}$ Finnish MAGIC Group: Finnish Centre for Astronomy with ESO, University of Turku, FI-20014 Turku, Finland ${ }^{26}$ Departament de Física, and CERES-IEEC, Universitat Autònoma de Barcelona, E-08193 Bellaterra, Spain ${ }^{27}$ Japanese MAGIC Group: Physics Program, Graduate School of Advanced Science and Engineering, Hiroshima University, 739-8526 Hiroshima, Japan ${ }^{28}$ Armenian MAGIC Group: ICRANet-Armenia at NAS RA, 0019 Yerevan, Armenia ${ }^{29}$ Croatian MAGIC Group: University of Split, Faculty of Electrical Engineering, Mechanical Engineering and Naval Architecture (FESB), 21000 Split, Croatia ${ }^{30}$ Croatian MAGIC Group: Josip Juraj Strossmayer University of Osijek, Department of Physics, 31000 Osijek, Croatia ${ }^{31}$ Japanese MAGIC Group: Department of Physics, Kyoto University, 606-8502 Kyoto, Japan ${ }^{32}$ Japanese MAGIC Group: Department of Physics, Tokai University, Hiratsuka, 259-1292 Kanagawa, Japan ${ }^{33}$ Saha Institute of Nuclear Physics, HBNI, 1/AF Bidhannagar, Salt Lake, Sector-1, Kolkata 700064, India ${ }^{34}$ Inst. for Nucl. Research and Nucl. Energy, Bulgarian Academy of Sciences, BG-1784 Sofia, Bulgaria ${ }^{35}$ Japanese MAGIC Group: Department of Physics, Yamagata University, Yamagata 990-8560, Japan ${ }^{36}$ Finnish MAGIC Group: Astronomy Research Unit, University of Oulu, FI-90014 Oulu, Finland ${ }^{37}$ Croatian MAGIC Group: Ruđer Bošković Institute, 10000 Zagreb, Croatia ${ }^{38}$ INFN MAGIC Group: INFN Sezione di Perugia, I-06123 Perugia, Italy ${ }^{39}$ INFN MAGIC Group: INFN Roma Tor Vergata, I-00133 Roma, Italy ${ }^{40}$ Japanese MAGIC Group: Department of Physics, Konan University, Kobe, Hyogo 6588501, Japan ${ }^{41}$ also at International Center for Relativistic Astrophysics (ICRA), Rome, Italy ${ }^{42}$ now at Department for Physics and Technology, University of Bergen, NO-5020, Norway ${ }^{43}$ now at University of Innsbruck ${ }^{44}$ also at Port d'Informació Científica (PIC), E-08193 Bellaterra (Barcelona), Spain ${ }^{45}$ now at Ruhr-Universität Bochum, Fakultät für Physik und Astronomie, Astronomisches Institut (AIRUB), 44801 Bochum, Germany ${ }^{46}$ now at Department of Astronomy, University of California Berkeley, Berkeley CA $94720{ }^{47}$ also at Dipartimento di Fisica, Università di Trieste, I-34127 Trieste, Italy 49 now at Laboratoire d'Annecy de Physique des Particules (LAPP), CNRS-IN2P3, 74941 Annecy Cedex, France ${ }^{50}$ also at INAF Trieste and Dept. of Physics and Astronomy, University of 
Bologna, Bologna, Italy 